\title{
SOME FEATURES OF THE MAGNETIZATION OF THIN LAYERS OF MAGNETIC FLUIDS WITH MAGNETIZED AGGREGATES IN MAGNETIC AND ELECTRIC FIELDS
}

\author{
D.V.Gladkikh, Yu.I.Dikansky, A.G.Ispiryan \\ North-Caucasus Federal University, Stavropol 355009, Russia \\ e-Mail: GladkikhDV@mail.ru
}

The paper presents results of the investigation of magnetization features of thin layers of differently based magnetic colloids with a well-developed system of magnetized aggregates, exposed to magnetic and electric fields. It has been found that the magnetization processes of volumetric samples and of thin layers differ. For thin layers of the investigated magnetic fluid, a dependence of the magnetization relaxation time on the layer thickness and also on the mutual orientation of the layer plane and external constant magnetic field is found. It is shown that an alternating electric field also affects the magnetic properties of a thin layer of magnetic colloid with magnetized aggregates.

Introduction. The magnetic properties of magnetic material can change significantly when its shape changes from volumetric to film-like. That is why thin layers and films of magnetic colloids (MC) could be of interest to investigate. Many theoretical and experimental studies address the problem of MC thin films [1-5] and consider mainly their (film) structural features under the action of magnetic and electric fields. However, there are practically no investigations which could compare the physical properties (in particular, magnetic) of film-like and volumetric samples. Also, no data have been published on the dependence of the physical properties of MC film-like samples on their thickness (except for the studies addressed in [6]). The kinetics of magnetization of such samples should significantly differ. The problem becomes much more topical when a magnetic colloid under study has a well-developed system of magnetized aggregates. In this case, under the action of external fields, some structural ordering of the dipoles could take place in a thin layer of such medium [7]. This, in turn, could significantly affect the magnetization processes and kinetics in these MC systems [8].

The structural ordering of magnetic colloids could be greatly affected by electric fields as well. However, their influence on the magnetization of the MC thin layers with magnetized aggregates has not been investigated so far.

Investigations of the thin layers of such magnetic colloids were earlier attempted in [6]. It was found that the magnetization processes in volumetric samples and in thin layers were different. For thin layers of the investigated magnetic colloid, the magnetization curves had a hysteretic character and the dependence of the remanent magnetization on the thickness of the layer was found.

In this paper, we present the results of the investigation of magnetization features of differently based magnetic colloids with a well-developed system of magnetized aggregates, determined by the parameters of the measuring cell in magnetic and electric fields.

1. Investigation objects and techniques. Two samples of magnetic colloids with well developed systems of magnetized aggregates were investigated. The first sample was a kerosene-based magnetic colloid with a volume concentration of the magnetic phase of $10.4 \%$. The average particle size was $12.1 \mathrm{~nm}$. Another sample was a magnetic colloid based on vacuum oil with a volume concentration 
of the magnetic phase of $8.3 \%$. We applied the method described in [9] to create magnetized aggregates in the investigated MC samples.

The measuring cell consisted of two rectangular glass plates. The thickness of the sample layer varied from 40 to $460 \mu \mathrm{m}$ and was determined by the thickness of the fluoroplastic films.

A cell made as a parallelepiped of $70 \times 20 \times 0.9 \mathrm{~mm}$ was used to study the magnetic susceptibility of magnetic fluid (MF) in an electric field. Two lateral wide walls were made of foil-coated fiberglass (foil inside). Between the electrodes there was a fluoroplastic film, the thickness of which was determined by the thickness of the sample layer. A magnetic colloid filled the cell and was in direct contact with the conducting walls. The electric field inside the cell was perpendicular to the plane of the layer.

For comparison, cylindrical tubes filled with the magnetic colloid under study were used as volumetric samples. The length of the tubes was $30 \mathrm{~cm}$, the internal diameter varied from 1 to $6 \mathrm{~mm}$.

The measuring cell was placed inside a flat measuring coil which was located in the central zone of the magnetizing system.

The magnetic susceptibility was measured by the bridge method. The real $\left(\chi^{\prime}\right)$ and imaginary $\left(\chi^{\prime \prime}\right)$ parts of the complex magnetic susceptibility were calculated from the variation of the inductance and $Q$-factor of the solenoid when a sample was introduced. Measurements were made using an LCR-817 immittance meter; the error in determining the effective value did not exceed $0.1 \%$ for the real part $\chi^{\prime}$ of magnetic susceptibility and of $0.3 \%$ for its imaginary part $\chi^{\prime \prime}$. Frequency dependences of the susceptibility were studied in the absence and in the presence of a steady uniform external magnetic field.

The external magnetic field $H_{0}$ was created by a cubic coil with a fivesectioned winding [10], with the winding ratio being $19: 4: 10: 4: 19$. The field non-uniformity at the place of location of the measuring cell windings did not exceed $0.3 \%$.

For thin layers, the frequency investigations of the magnetic susceptibility dependence were performed for three configurations of magnetic fields (Fig. 1): i) the external magnetic field was orthogonal to the measuring field and to the layer plane (Fig. 1a); ii) the external magnetic field was orthogonal to the measuring field and parallel to the layer plane (Fig. 1b); iii) the external magnetic field was parallel to the measuring field and to the layer plane (Fig. 1c).

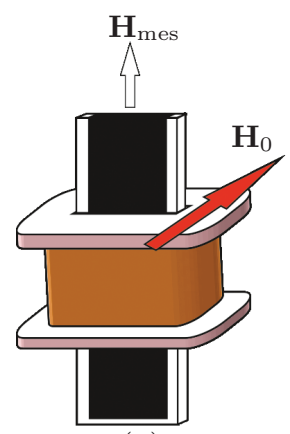

(a)

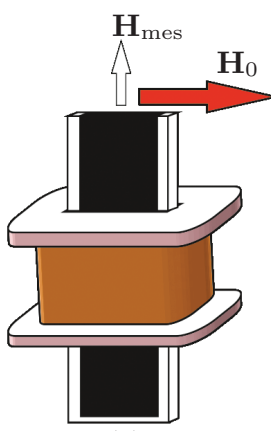

(b)

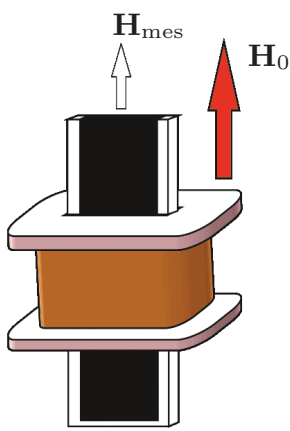

(c)

Fig. 1. Mutual orientation of external steady and measuring magnetic fields in experiment. 
Some features of the magnetization of thin layers of magnetic fluids with ...

2. Results and discussion. In order to continue the initiated studies [6], frequency investigations of the complex magnetic susceptibility of kerosene-based magnetic colloids with magnetized aggregates were carried out with volumetric cylinder-shaped samples and with thin layer samples. For comparison, similar studies of a homogeneous sample of the same volume concentration were conducted.

For all investigated samples (cylindrical and thin layers) it has been found that the frequency dependences of the imaginary part of the complex magnetic susceptibility achieve a maximum at certain frequency even in the absence of the external magnetic field, which allowed calculating the time of relaxation.

For the volumetric (cylinder-shaped) samples, it has been found that the relaxation time increases with the decrease of the diameter of the tube with the sample both in the case of parallel alignment of the alternating measuring and external steady magnetic fields and in the case of their mutually perpendicular orientation. For the MC thin layer samples with magnetized aggregates, a similar situation took place, i.e. the time of relaxation increased with the reducing thickness of the layer. However, the character of this dependence differs for different mutual orientations of the layer plane and external magnetic fields (Fig. 2). When the external magnetic field was aligned parallel to the layer (Figs. $2 b, c$ ), the layer thickness reduction resulted in monotonic increase of the relaxation time. When the external magnetic field was orthogonal to the layer plane and to the measuring field (Fig. 2a), the relaxation time vs. layer thickness dependence demonstrates a maximum, the position of which does not depend on the magnitude of the external magnetic field.
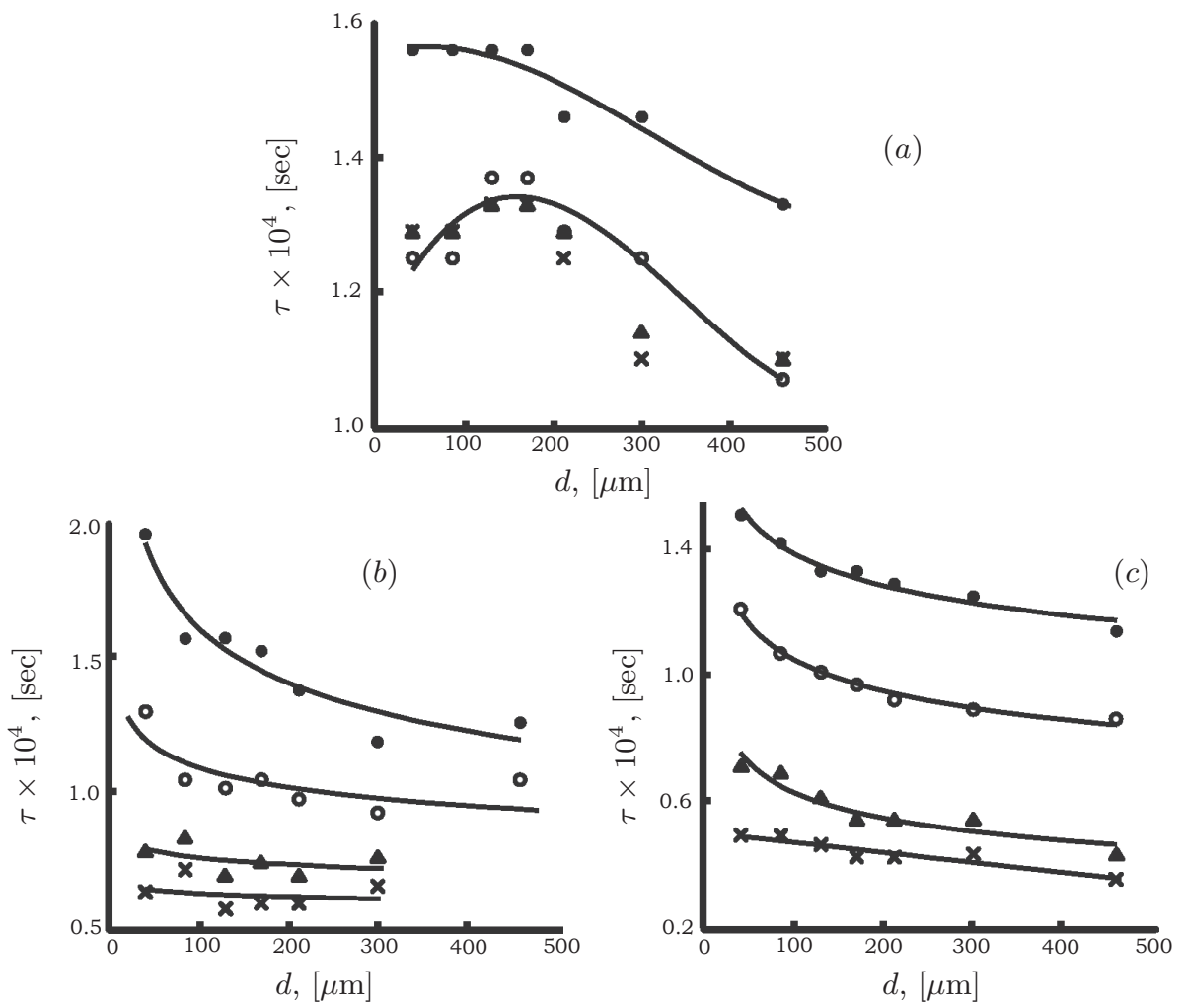

Fig. 2. Relaxation time vs. layer thickness for various orientations of external and measuring magnetic fields. $\bullet-H=0 \mathrm{Oe} ; \circ-H=10.8 \mathrm{Oe} ; \boldsymbol{\Delta}-H=21.6 \mathrm{Oe} ; \times-$ $H=27$ Oe. 


\section{D.V. Gladkikh, Yu.I.Dikansky, A.G. Ispiryan}

For comparison, the frequency dependences of the complex magnetic susceptibility for thin layers of the MC homogeneous sample without aggregates were studied as well. It is found that the frequency dependences of such sample have no maximum in the entire studied range of frequencies and magnitudes of the external magnetic fields. Hence, it was not possible to find out experimentally the dependence of the relaxation time for thin layers of a homogeneous magnetic colloid. As a result, we have a theoretical dependence of the relaxation time on the layer thickness for a homogeneous MF sample of similar dimensions when the external magnetic field is perpendicular to the measuring field and to the plane of the layer (Fig.2a).

The time of relaxation was defined by the formula [11] as

$$
\begin{gathered}
\tau=\tau_{B}\left[1-\frac{1}{6}\left(\frac{\mu_{0} m H^{\prime}}{k T}\right)^{2}\right], \\
H^{\prime}=H_{0}-N M, \\
N^{-1}=N_{\mathrm{c}}+0.144 \frac{a}{b} \frac{d}{\sqrt{b d}} \mathrm{e}^{-0.025 a / \sqrt{b d}} .
\end{gathered}
$$

Here, $N_{\mathrm{c}}$ is the demagnetizing factor of a similar cylinder with the same crosssectional area as a rectangular sample, $H_{0}$ is the external magnetic field strength, $\mu_{0}$ is the magnetic constant, $m$ is the magnetic moment of colloid particles, $M$ stands for the magnetization, $a, b, d$ are, respectively, the length, the width and the thickness of the sample.

For comparison, Fig. 3 shows the obtained experimental (for a sample with magnetized aggregates) and theoretical (for a homogeneous sample) dependences of the relaxation time on the layer thickness. As seen in the figure, the dependence of the relaxation time on the thickness of the layer for a homogeneous sample has a monotonically decreasing character when exposed to an external magnetic field. It can be concluded that the maximum of the relaxation time dependence is due to the presence of magnetized aggregates and, apparently, to the features of their spatial orientation in a thin layer under the action of the mutually perpendicular alternating and steady magnetic fields.

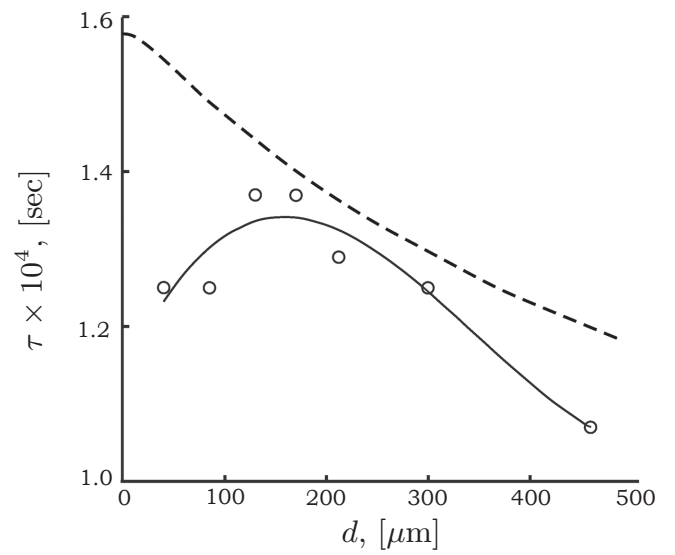

Fig. 3. Relaxation time vs. layer thickness under additional action of an external steady magnetic field $H=10.8$ Oe directed perpendicular to the layer plane (Fig. $2 a$ ): (-) experimental curve, (-- ) theoretical curve. 
Some features of the magnetization of thin layers of magnetic fluids with ...

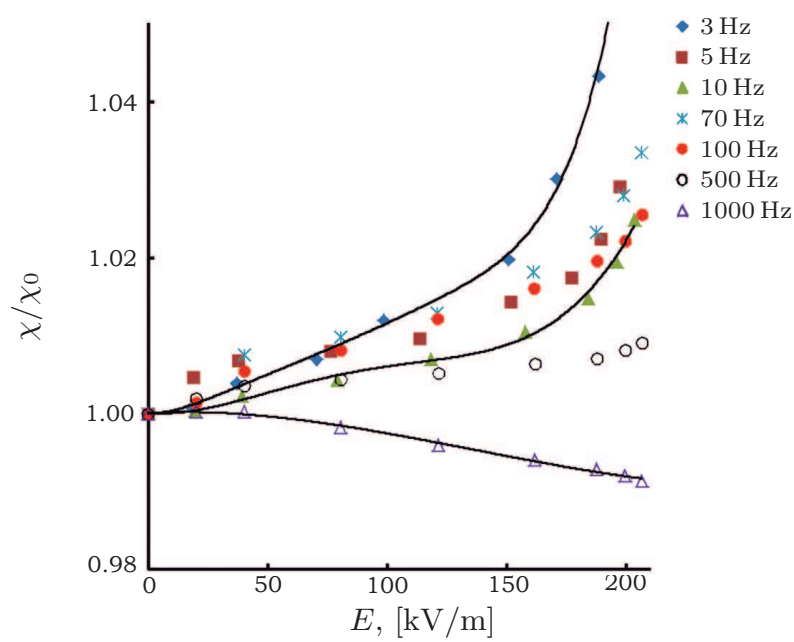

Fig. 4. Relative magnetic susceptibility vs. effective value of the intensity of the AC electric field of different frequency.

It was found that the action of an alternating electric (AC) field also resulted in a change of the magnetic properties of the $\mathrm{MC}$ thin layer. In the experiments, a vacuum oil-based magnetic colloid with a well-developed system of magnetized aggregates was investigated. Fig. 4 illustrates the dependences of the relative magnetic susceptibility ( $\chi_{0}$ is the magnetic susceptibility with the electric field intensity $E=0 \mathrm{kV} / \mathrm{m}$ ) on the effective value of the intensity of the electric field of different frequency. The figure demonstrates that at low frequencies of the AC field $(3-100 \mathrm{~Hz})$, the relative magnetic susceptibility grows monotonically with increasing electric field intensity. At frequencies of about $500 \mathrm{~Hz}$, the relative magnetic susceptibility practically ceases to vary with the field increase (the variations do not exceed 1\%). At large frequencies of $1000 \mathrm{~Hz}$, a monotonic decrease of the relative magnetic susceptibility was observed.

Additional influence by the steady magnetic field aligned with the MC sample layer and orthogonal to the electric field resulted in a significant alteration of the dependence of the magnetic susceptibility on the electric field. Fig. 5 displays the dependences of the relative magnetic susceptibility on the effective value of the intensity of the $\mathrm{AC}$ field of different frequency under the additional action of the steady magnetic field $H=4.8 \mathrm{Oe}$. In this case, the relative magnetic susceptibility value monotonically decreases with the rise of the electric field intensity for all investigated frequencies. Moreover, as the figure demonstrates, with the increasing frequency of the $\mathrm{AC}$ field the relative magnetic susceptibility varies less.

It was suggested that the discovered peculiarities of the magnetic susceptibility of the MC sample under investigation were determined by the processes of structural ordering in the system of magnetized aggregates when the sample was exposed to the AC and steady magnetic fields. The optical observation revealed that when the sample was affected only by the AC field of low frequency $(3-100 \mathrm{~Hz})$, the aggregates, remaining separated, were driven into rotary motion whose intensity grew with increasing field strength. This likely determines the increase of the relative magnetic susceptibility (see Fig. 4, $3 \mathrm{~Hz}$ curve).

The increase of the AC field frequency led to the decrease of the rotary motion frequency, which resulted in less significant variations of the magnetic susceptibility (Fig. 4, 10-100 Hz curve). With frequencies exceeding $500 \mathrm{~Hz}$, partial agglomer- 


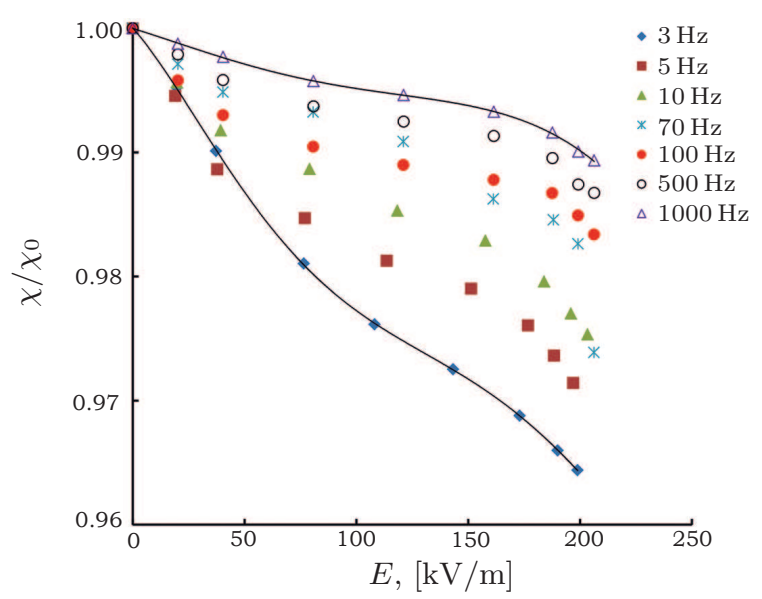

Fig. 5. Relative magnetic susceptibility vs. effective value of the intensity of the AC electric field of different frequency under additional action of the steady magnetic field $H=4.8$ Oe.

ation of the aggregates into large elongated structures was observed which, with the increase of the AC field strength, began to align with the electric field. Accordingly, they should be positioned orthogonally to the measuring magnetic field in the measuring cell to increase hence the effective demagnetizing factor in the direction of the measuring field, which must result in decrease of the magnetic susceptibility and which was observed in the experiment (Fig. 4, $1000 \mathrm{~Hz}$ curve).

Under the additional impact of the steady magnetic field directed orthogonally to the electric field, the magnetized aggregates started to agglomerate into structures stretched out along the magnetic field. The action of the AC field resulted in periodic detachment of smaller groups, consisting of several magnetized aggregates and elongated, as before, along the magnetic field, from these large agglomerates.

After detachment, these small aggregates were almost at once attracted to the neighboring agglomerates. The intensity of such detachments and attractions was observed to be dependent on the $\mathrm{AC}$ field frequency, i.e. with increasing field frequency this intensity decreased. These processes were also influenced by the value of the steady magnetic field intensity. At large strengths of the steady magnetic field, all aggregates were grouped into agglomerates stretched out along the magnetic field; the configuration of these agglomerates was not practically affected by the electric field.

3. Conclusion. The performed studies have shown that the processes of magnetization of volumetric samples of magnetic colloids with magnetized aggregates and of their thin layers differ. It has been found that for a thin layer the dependence of the magnetization relaxation time depends both on the thickness of the layer and on the orientation of the external magnetic field with respect to the plane of the layer.

In addition, it is found that the $\mathrm{AC}$ field action also results in variation of the magnetic properties of thin layers of magnetic colloid with magnetized aggregates. The observed change in magnetic susceptibility of the thin layer could be explained by the processes of structural transformation in the system with magnetized aggregates, which turns out to be depended on the frequency of the electric field and also on the additional action of the steady magnetic field directed perpendicular to the electric field. 
Some features of the magnetization of thin layers of magnetic fluids with ...

4. Acknowledgements. This work was supported by The Ministry of Edu-

cation and Science of the Russian Federation and by the Russian Foundation for Basic Research (RFBR) (project No. 18-03-00279).

\section{References}

[1] H. Wang, Y. Zhu, C. Boyd, W. Luo, A. Cebers, and R.E. Rosensweig. Periodic branched structures in a phase-separated magnetic colloid. Phys. Rev. Let., vol. 72 (1994), no. 12, pp. 1929-1932.

[2] J. Liu, E.M. Lawrence, A. Wu, M.L. Ivey, G.A. Flores, K. Javier, J. Bibette, AND J. Richard. Field-induced structures in ferrofluid emulsions. Phys. Rev. Let., vol. 74 (1995), no. 14, pp. 2828-2831.

[3] J.M. Tavares, J.J. Weis, and M.M. Telo da Gama. Quasi-two-dimensional dipolar fluid at low densities: Monte Carlo simulations and theory. Phys. Rev. E, vol. 65 (2002), p. 061201.

[4] T. KristF, I. Szalai. Magnetic properties in monolayers of a model polydisperse ferrofluid. Phys. Rev. E, vol. 72 (2005), p. 041105.

[5] R. Haghgooie and P.S. Doyle. Transition from two-dimensional to threedimensional behavior in the self-assembly of magnetorheological fluids confined in thin slits. Phys. Rev. E, vol. 75 (2007), p. 061406.

[6] Yu.I. Dikansky, D.V. Gladkikh, A.Yu. Shevchenko, A.A. SidelniKOV. Magnetization features of thin layers of magnetic fluids with a welldeveloped system of magnetized aggregates. Magnetohydrodynamics, vol. 50 (2014), no. 1, pp. 27-33.

[7] D.V. Gladkikh, Yu.I. Dikansky, A.A. Kolesnikova. Structural transformations in a thin layer of magnetic colloid with magnetized aggregates exposed to an elliptically polarized rotating magnetic field. Magnetohydrodynamics, vol. 52 (2016), no. 3, pp. 319-332.

[8] Yu.I. Dikansky, D.V. Gladkikh, S.A. Kunikin, A.A.Zolotukhin. Magnetic ordering in colloidal systems of single-domain particles. Magnetohydrodynamics, vol. 48 (2012), no. 3, pp. 493-501.

[9] Yu.I. Dikansky, Zh.G. Veguera, R.G. Zakinyan, O.A. Nechaeva, D.V. GladkikH. Coloidal Journal, vol. 67 (2005), no. 2, pp. 134-139 (in Russian).

[10] I.I. KIfER. Testing of Ferromagnetic Materials (Gosenergoizdat, MoscowLeningrad, 1962), 544 p. (in Russian).

[11] M.I. Shliomis. Magnetic fluids. Advances of Soviet Physics, vol. 17 (1974), no. 2, pp. 153-170 (in Russian).

Received 27.12.2017 